\title{
REVIEW
}

\section{High-Dose Capsaicin for the Treatment of Neuropathic Pain: What We Know and What We Need to Know}

Nurcan Üçeyler • Claudia Sommer

To view enhanced content go to www.paintherapy-open.com

Received: April 15, 2014 / Published online: July 29, 2014

(C) The Author(s) 2014. This article is published with open access at Springerlink.com

\section{ABSTRACT}

Neuropathic pain is a frequent and disabling condition with diverse underlying etiologies and is often difficult to treat. Systemic drug treatment is often limited in efficacy. Furthermore, adverse effects may be a limiting factor when trying to reach the necessary dose. Analgesics that can be applied topically have the potential to largely overcome this problem. They may be of particular advantage in localized neuropathic pain syndromes such as postherpetic neuralgia or small fiber neuropathy. Capsaicin, the pungent component of chili peppers, is a natural ligand of the transient receptor potential vanilloid 1 channel and has long been used as topically applicable cream with concentrations of 0.025 to $0.075 \%$. In 2009, a high-concentration transdermal capsaicin 8\% patch $\left(\right.$ Qutenza $^{\circledR}$; Acorda Therapeutics, Inc., Ardsley, NY, USA;

Electronic supplementary material The online version of this article (doi:10.1007/s40122-014-0027-1) contains supplementary material, which is available to authorized users.

\section{N. Üçeyler · C. Sommer $(\bowtie)$}

Department of Neurology, University of Würzburg, Josef-Schneider-Str. 11, 97080 Würzburg, Germany e-mail: sommer@uni-wuerzburg.de
Astellas Pharma Europe Ltd., Chertsey, Surrey, UK) was introduced for the treatment of peripheral neuropathic pain syndromes other than of diabetic origin in adults. It has since been widely used in diverse neuropathic pain disorders. In this review article, we summarize current knowledge on Qutenza, its advantages and problems, and expose unmet needs.

Keywords: Analgesia; Capsaicin; Neuropathic pain; Qutenza; Transient receptor potential vanilloid 1 (TRPV1)

\section{NEUROPATHIC PAIN}

Neuropathic pain is caused by a lesion or disease of the somatosensory nervous system [1] and affects an estimated $8 \%$ of the general population [2], leading to severe impairment and reduction of health-related quality of life. The peripheral nervous system is more frequently the source of neuropathic pain rather than the central nervous system. Examples for peripheral neuropathic pain syndromes include postherpetic neuralgia (PHN), painful diabetic neuropathy, human 
immunodeficiency virus (HIV)-associated neuropathy, or chemotherapy-induced neuropathic pain. Phantom limb pain after amputation is considered to have a peripheral and central component. Spinal cord injury pain or pain due to cerebral infarction would be examples for centrally induced neuropathic pain conditions. Peripheral neuropathic pain is mostly localized in the area supplied by the affected nerves and is reported to be of burning, stabbing, or electrifying character. Additional symptoms are plus symptoms such as hyperalgesia (i.e., increased pain upon application of painful stimulus), allodynia (i.e., pain upon application of painless stimulus), painless paresthesias, or painful dysesthesias, and minus symptoms that include hypoesthesia and hypoalgesia [3]. While neuropathic pain and additional symptoms initially may be of episodic character, in the majority of cases pain becomes permanent and chronic in the long term.

The causes of neuropathic pain are diverse. From the clinical point of view, trauma, hemorrhage, ischemia, inflammation, or metabolic alterations are some examples of how the central and the peripheral parts of the somatosensory nervous system can be impaired. However, this small and selective list of possibilities already implies that the pathophysiological mechanisms underlying neuropathic pain are manyfold. These mechanisms are still incompletely understood despite intensive research.

Pathological ion channel activity is of particular importance when discussing neuropathic pain pathophysiology. Different subgroups of ion channels are critically involved in neuropathic pain development via ectopic discharges and sensitization. The family of voltage-gated sodium channels $(\mathrm{NaV})$ is an outstanding example since the discovery of mutations in the gene of Nav1.7 as the molecular basis of erythromelalgia first opened the avenue of genetic pain research [4]. Another family of voltage-gated ion channels that has a crucial role in neuropathic pain is the transient receptor potential (TRP) family. The TRP channels regulate action potential firing frequencies by gating neuronal transmembrane ion influx and modulate the sensitivity of afferent somatosensory neurons [5]. Another factor inducing and maintaining neuropathic pain is the dysfunctional effect and imbalance of algesic and analgesic mediators like cytokines or chemokines during neuro-immune interactions in the peripheral and central nervous system [6]. $\mathrm{NaV}$ as well as TRP channels are modulated by these mediators $[7,8]$.

So far, the best studied member of the TRP is the transient receptor potential vanilloid 1 (TRPV1) channel [9]. This non-selective cation channel is highly expressed on thinly myelinated A-delta fibers and unmyelinated C fibers (nociceptors) and is in particular permeable to calcium ions. TRPV1 activation typically leads to burning pain sensation and heat hyperalgesia [9]. Numerous TRPV1 activators are known to date that include high temperatures $\left(>42^{\circ} \mathrm{C}\right)$, acidic $\mathrm{pH}$, and the pungent ingredient of hot chili peppers, capsaicin, which is a natural ligand of TRPV1 and makes this channel a delicate candidate for pain research.

\section{CURRENT PHARMACOLOGICAL TREATMENTS OF NEUROPATHIC PAIN AND UNMET NEEDS}

Treatment of neuropathic pain follows national $[10,11]$ and international guidelines $[12,13]$ that broadly overlap with regard to recommendations. In most guidelines, firstline therapy is the use of oral drugs such as 
tricyclic antidepressants (e.g., amitriptyline), anticonvulsants including calcium channel blockers (e.g., gabapentin, pregabalin), and selective serotonin and noradrenalin reuptake inhibitors (e.g., duloxetine). In the case of localized pain, topical lidocaine can be applied as well as capsaicin cream or patch. If patients do not respond or have mixed pain (i.e., neuropathic pain plus nociceptive pain) the use of opioids can be considered [10].

Oral medication is used by the majority of patients suffering from neuropathic pain; however, only one-third of these patients seem to achieve satisfying pain relief [14]. Thus, the main problem with oral drugs is the lack of efficacy in a large proportion of patients even after intake of a sufficient dosage, changing to alternative drugs, and when used in combination. In addition, the occurrence of systemic side effects such as weight gain, xerostomia, dizziness, nausea, or cognitive impairment hampers acceptance. The fact that oral medication also needs individual titration and regular intake on a daily basis is an additional limitation reducing flexibility in life, especially for young patients. Drug-drug interactions may also constrain the already limited treatment options, especially in elderly patients with comorbidities. In this context, drug dosage needs to be adapted if renal or hepatic impairment is present. The effect of oral analgesic drugs also starts late; for some drugs an intake period of $6-8$ weeks at the maximum dose is needed before drug efficacy can be judged. In localized neuropathic pain states, topically applicable lidocaine and ketamine, as well as low-dose capsaicin cream (0.025-0.075\%), are in use. However, these require regular administration and also bear less hazardous but inconvenient disadvantages (e.g., potential contamination of hands during application or clothes afterwards).
Thus, despite a major effort to improve and facilitate oral analgesics there are still several challenges to be taken to meet the needs of patients with neuropathic pain syndromes [15]. One key obstacle is the lack of knowledge on the precise mechanisms that underlie the different types of neuropathic pain and that drugs are chosen mostly with regard to neuropathic pain in general without differentiation. Neuropathic pain, in general, covers many diverse entities that are of such a different pathophysiological background that a "one-drug-good-for-all" approach is condemned to failure. A second problem is that currently available analgesic pharmaceuticals are not exclusively selective for one target. Unselective drug choice (i.e., treating neuropathic pain in general) and molecularly relative unselective drugs (i.e., binding to different targets) are the major reasons that lower drug efficacy and tolerability. Therefore, the transdermal capsaicin 8\% patch Qutenza ${ }^{\circledR}$ (Acorda Therapeutics, Inc., Ardsley, NY, USA; Astellas Pharma Europe Ltd., Chertsey, Surrey, UK) has been an important addition to the treatment options in neuropathic pain conditions [16].

\section{HIGH-DOSE CAPSAICIN}

\section{Mechanism of Action}

Transient receptor potential vanilloid 1 is selectively expressed in nociceptors. The distal endings of cutaneous nociceptors are present in the epidermis and are thus accessible to local treatment. The high-concentration transdermal 8\% capsaicin patch Qutenza releases capsaicin into the skin, which can then act on TRPV1 receptors on the nociceptor terminals. This leads to an initial over-excitation of these nerve fibers, which is perceived as burning pain by patients. After this initial excitation, 
the axons are believed to be "defunctionalized", that is, to be less sensitive to external stimuli and also to cease any spontaneous activity that may have been present. Morphologically, intraepidermal nerve fiber endings disappear after capsaicin application, which can be assessed using immunohistochemistry and the panaxonal marker protein gene product (PGP) 9.5 which is routinely used to visualize intraepidermal nerve fibers [17]. In the case of Qutenza, these nerve fiber endings recover after 24 weeks, at least in healthy volunteers [18]. Investigations in patients with pain states treated with capsaicin have not been performed so far. Thus, several questions remain, such as a potential correlation between fiber density and pain intensity and the phenotype of the regenerating fibers with regard to their channel repertoire. Also, whether this disappearance and recovery of immunostaining for PGP 9.5 reflects true degeneration and regeneration or repressed production of the antigen detected in the immunofluorescence, is as yet unknown. Another potential mechanism is based on the interaction of TRPV1 with $\beta$-tubulin. TRPV1 activation leads to a microtubule disassembly in vitro by direct action and supported by the axonal calcium ion overload via excessive ion influx [19]. Whether Qutenza has a similar effect in vivo remains to be proven.

One apparent paradox is that neuropathic pain states are usually associated with a loss of intraepidermal nociceptor terminals [20, 21]. If these target fibers are gone, where would a TRPV1 agonist bind? The most favored hypothesis in this context is that the remaining nociceptors become hyperactive and hypersensitive on the basis of alterations in TRPV1 channel activity and expression. There is evidence for an increase in TRPV1 channel quantity on inflamed nociceptors [22] and also for the sensitization of these nociceptors by local algesic inflammatory mediators and growth factors [23, 24].

There are several other unsolved questions. It is unclear why some patients do respond to Qutenza treatment and others do not. If Qutenza leads to nociceptor defunctionalization or degeneration, then all patients suffering from peripheral neuropathic pain should experience pain relief. Here, diversity of neuropathic pain pathophysiology and mechanisms is important. Obviously, TRPV1-mediated pain is not responsible for all neuropathic pain states. This is also underscored by the fact that some patients develop a skin flush upon patch application which can be associated with severe additional burning patch-pain for days to weeks and some do not. Interestingly, the development of such a patch-pain does not predict treatment response [41]. No data are available about how deep capsaicin from the Qutenza patch penetrates the different skin layers and how much capsaicin reaches the nociceptors. Skin penetration studies with Qutenza are ongoing, as with other novel formulations [25]. It is also not known whether capsaicin acts on peripheral nerve TRPV1 channels only or if an action, for example, on keratinocyte TRPV1 channels, also plays at least a modulatory role [26]. The pharmacokinetics of capsaicin in the skin are still under investigation (J. Wohlrab, personal communication, January 2014).

\section{Dose and Administration, and Benefits of Localized Rather Than Systemic Analgesia}

The application of the transdermal capsaicin 8\% patch Qutenza containing $179 \mathrm{mg}$ capsaicin needs to be performed at a medical center as special precautions are needed [27]. First, the 
area that needs to be treated has to be determined and marked by the treating physician or the applying nurse. Afterwards, the skin is cleaned and lidocaine gel may be applied to reduce patch-induced pain; alternatively, patients may take oral analgesics (e.g., tramadol) prior to Qutenza application (see below). Thereafter, the Qutenza patch is placed on the affected region for $30 \mathrm{~min}$ if the feet are treated or for $60 \mathrm{~min}$ for any of the other approved body regions. After this time, the patch is removed and the affected skin area is cleansed. The effect of the Qutenza patch starts within days and analgesia can be achieved for at least 12 weeks. After this time treatment can be repeated.

The major advantages of the localized treatment are that potential systemic side effects of Qutenza, comprising hypertension, first-degree atrioventricular block, coughing, or nausea, occur very rarely. Side effects that are usually associated with the intake of analgesics like cognitive impairment or drowsiness are absent. This is of particular relevance for young patients who work and drive cars. For elderly patients who also need to take other drugs, the local application of transdermal Qutenza is an advantage since no drug-drug interactions will occur.

\section{Clinical Trial Data That Led to its Launch, Including the Recent EC Approval for Expanded Treatment Options}

In 2009, the European Medicines Agency approved the use of Qutenza for the treatment of peripheral neuropathic pain other than of diabetic origin in adults as a monotherapy or in combination with other analgesic drugs [28]. The approval was based on data from randomized, double-blind, placebo controlled studies in patients with PHN [29-32] and painful HIV-associated neuropathy [33, 34] on the efficacy and safety of transdermal $8 \%$ capsaicin versus a control patch with low-dose capsaicin (0.04\%).

In one multicenter, randomized-controlled trial (RCT), 206 patients with PHN were treated with transdermal $8 \%$ capsaicin and $42 \%$ of them reported $a \geq 30 \%$ reduction in pain intensity versus $32 \%$ in patients treated with the placebo patch [29]. Similar results were reported by others after application of the $8 \%$ capsaicin patch in 194 patients [31]. In another RCT, 32 patients with PHN were randomized to be treated with transdermal $8 \%$ capsaicin; a decrease in pain intensity ratings of approximately $30 \%$ in the verum group compared to the control group was reported [30]. The treatment of 200 patients with PHN with $8 \%$ capsaicin patch led to a reduction of the mean percentage of pain ratings that was greater than in the placebo group [32]. In 225 patients with painful HIV-associated neuropathy, transdermal $8 \%$ capsaicin led to a reduction in pain ratings in $23 \%$ of patients on verum versus $11 \%$ of patients on placebo [34]. In another study this effect could not be confirmed [33]. Taken together one study [33] out of six was negative on the primary outcome with the capsaicin $8 \%$ patch, while in the other five studies more patients reported a positive effect when treated with the high-concentration patch compared to the low concentrated patch, as also stated in a recent Cochrane review [35]. Table 1 summarizes the major characteristics of the reported studies [29-34].

Particularly when comparing data of the verum (high-dose) and control (low-dose) patch in the reported studies, it is apparent that a low-dose capsaicin patch may also have a notable analgesic effect. In the majority of the studies summarized in Table 1 the difference in analgesic efficacy between the high and the 


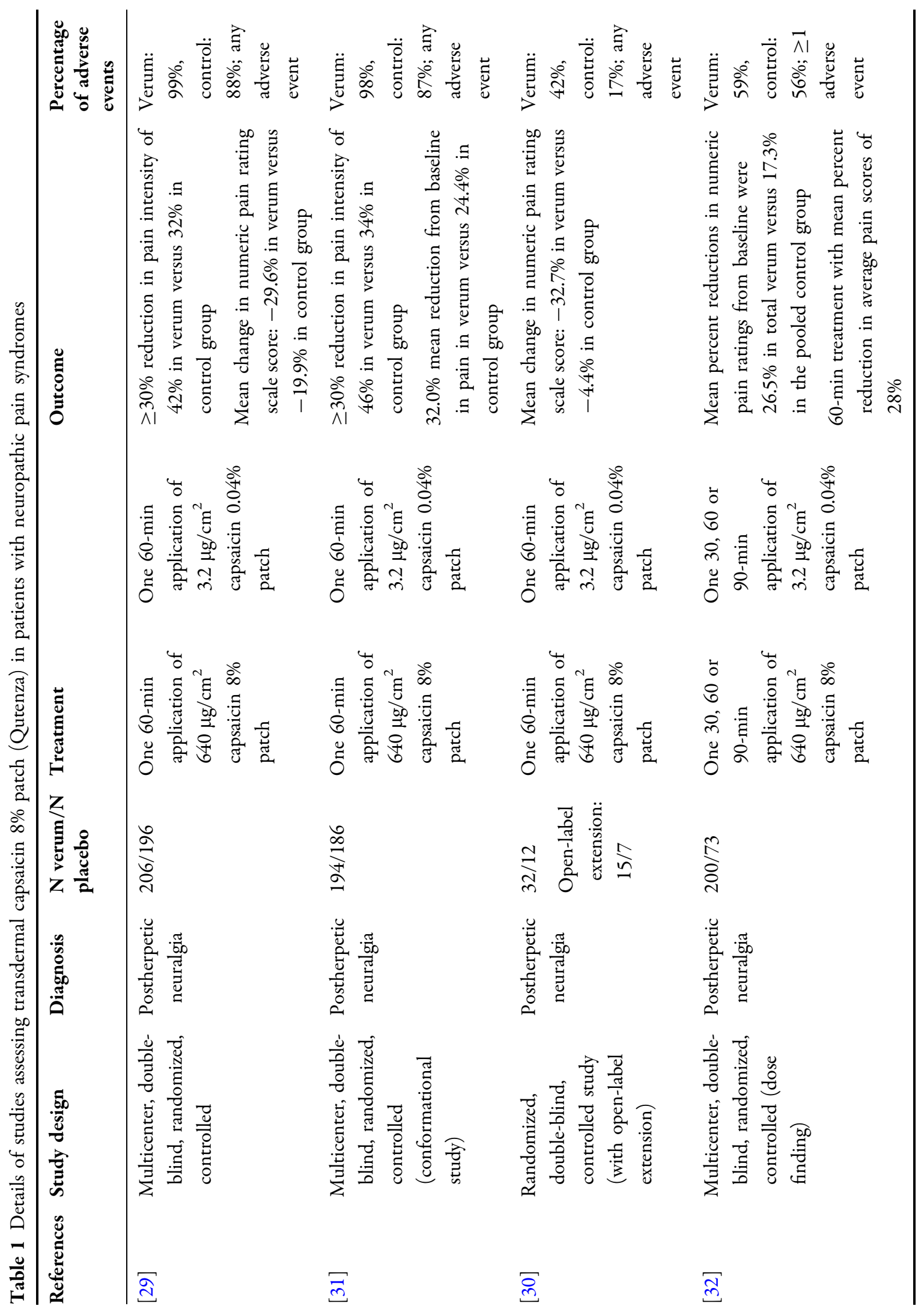




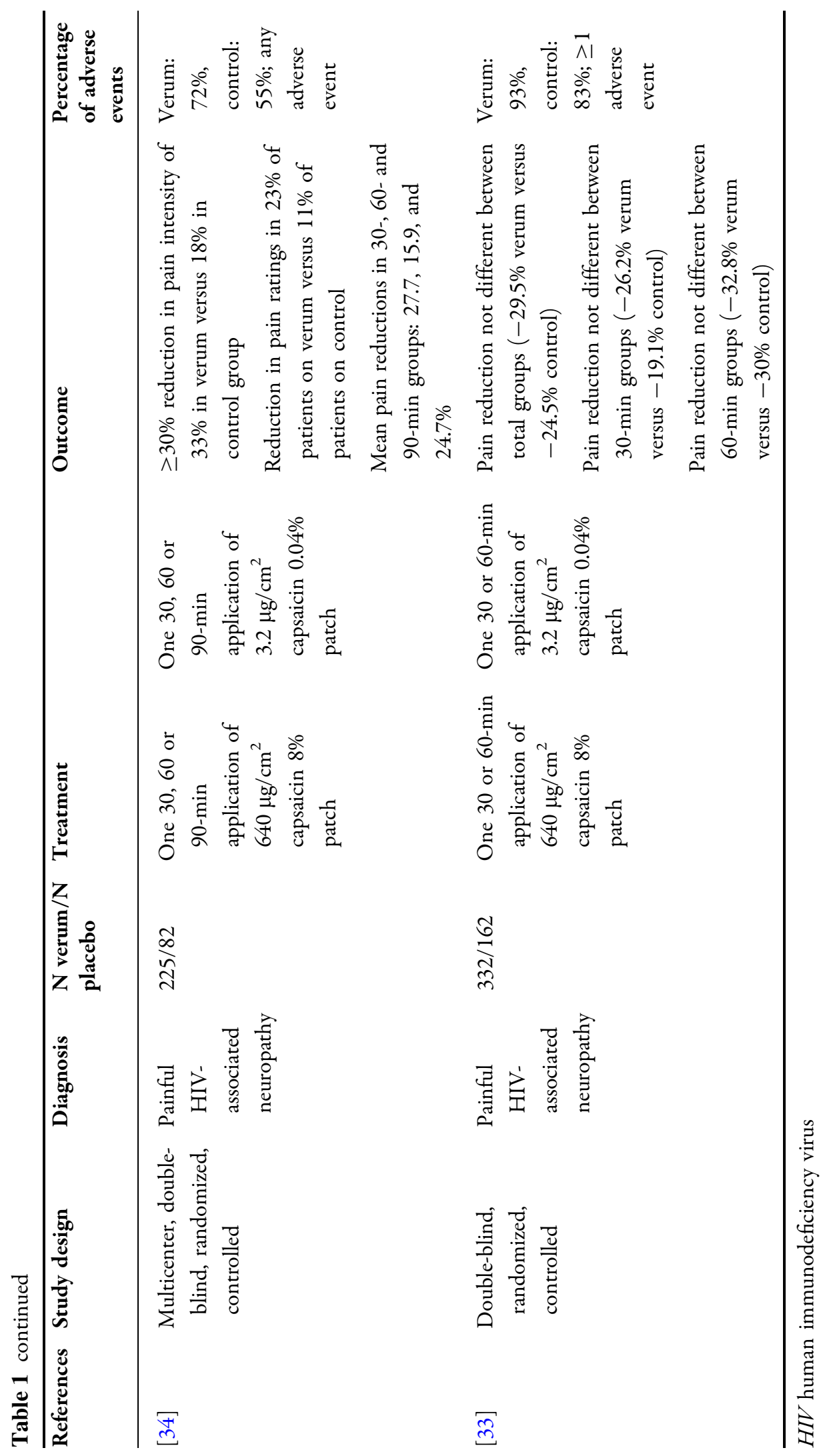


low-dose patch was less than $15 \%$. Side effects were reported inhomogenously in the respective studies, however, while high-dose capsaicin caused a higher percentage of adverse events, the control patch also led to similar results.

In 2013, an expanded pre-treatment procedure was approved by the European Commission based on the results of a study investigating 122 patients who received either the already approved topical lidocaine pretreatment before Qutenza application or tramadol tablets [36]. Both pre-treatment regimes proved to be equally effective and can now be used in clinical practice.

\section{HOW QUTENZA FITS INTO TREATMENT REGIMENS IN THE REAL WORLD}

In clinical practice, patients with neuropathic pain conditions are treated with oral pharmacological drugs as first-line therapy as recommended by national and international guidelines. However, not all patients treated with oral analgesic drugs experience pain relief to a satisfying extent. The consequence is that drug dosage is increased. If this measure also fails to reduce pain, the medication is changed to a drug from a different group, as detailed above, or combination pharmacotherapy is installed. During this mostly exhausting phase of trial and error, the outcome may be biased by decreasing patient compliance. The experience of ineffective drugs with unpleasant adverse effects reduces the motivation of the patient and sometimes may also lead to catastrophizing. The inconvenience of obligatory daily drug intake is in particular difficult to accept for young and active patients. In this situation, Qutenza may be an alternative for the treating physician and the patient. The novel application and the fact that treatment is episodic are attractive features that are very well accepted by the patients. Qutenza can then be used as monotherapy or in combination with oral analgesic drugs that may, however, be reduced in dosage. This is a good addition to the overall small palette of possibilities for noninterventional pharmaceutical analgesic treatment in neuropathic pain conditions.

\section{Current Experience}

Several studies have been published after the approval of Qutenza assessing the efficacy and safety in patients with PHN and HIV-associated painful neuropathy. A meta-analysis including 1,120 patients with PHN and 338 with HIVassociated neuropathy favored Qutenza over the low-dose capsaicin patch when assessing the number of patients with $30 \%$ pain reduction as primary outcome [37]. In these trials, overall $44 \%$ of the PHN and $41 \%$ of the HIV-associated neuropathy patients had a $30 \%$ response. Complete pain relief $2-12$ weeks after treatment was reported in $11 \%$ of patients with PHN and $7 \%$ of patients with HIV-associated neuropathy [38]. It took a mean time of 3.4 days for PHN patients and of 6.5 days for HIVneuropathy patients to respond. The mean duration of response after the first Qutenza treatment was 5 months [38]. In the largest non-interventional study so far, QUEPP (Qutenza-safety and effectiveness in peripheral neuropathic pain), the effect of a single Qutenza application was assessed over a period of 3 months in 1,044 patients with neuropathic pain other than of diabetic origin [39]. The authors reported that $43 \%$ of patients had $30 \%$ pain relief and 24\% experienced 50\% pain relief. Also, pain intensity and the frequency of pain attacks declined and 
additional intake of analgesic drugs was reduced. In this study [39], pre-treatment pain duration was negatively correlated to the effectiveness of Qutenza; however, there have been conflicting data depending on pain etiology [31, 40, 41].

Another question is how to determine who are responders and who are non-responders of Qutenza treatment. Efforts have been made to identify predictors of a positive Qutenza response. A post hoc analysis of the clinical trial data showed that efficacy of lidocaine pretreatment and a high pretreatment pain score variability were important predictors [42]. In a multi-center open study with multivariate analysis, time of disease was a major predictor, with a history of pain of less than 6 months being a positive predictor for a response to Qutenza [41]. In this context, effective lidocaine pretreatment and a higher pre-treatment pain score variability were found as potential predictors of good treatment response: older age and longer duration of pre-treatment pain were predictors for poorer outcome after Qutenza treatment [41, 42]. One critical aspect, however, is that a high efficacy of the control patch $(0.04 \%)$ was also reported [33, 37, 42].

Qutenza is mostly well tolerated. The major side effects are at site erythema, burning pain, and itch upon patch application that may last for days to weeks [35]. Currently no predictors are known for who will develop patch-pain. Also, no correlation exists so far with regard to patch-pain and analgesic effect of Qutenza.

\section{SUMMARY AND OUTLOOK}

Neuropathic pain is a major challenge due to chronification and low treatment response. The non-interventional pharmacological treatment options used so far are effective only in subgroups of patients and are mostly afflicted with intolerable side effects. Topically applicable substances are of need, especially in focal neuropathic pain conditions. Qutenza is therefore a valuable addition to the palette of analgesic options against neuropathic pain in adults. The application is easy and mostly well tolerated and leads to pain relief in a subset of patients.

The aim is to further improve and facilitate the Qutenza application so that, for example, it might be applied by the patients themselves. A further reduction in local side effects would be desirable. Further studies are underway to investigate the efficacy and safety of Qutenza in other peripheral neuropathic pain states including those related to diabetes. There are no studies about pain relief by Qutenza in children. Although no data are available on the prevalence of neuropathic pain in children, being able to use Qutenza in pediatric patients with localized neuropathic pain might be a worthwhile goal with regard to the general reluctance to give systemic analgesics in child pain management. Data on potential biomarkers that can be used as potential predictors of treatment response would be useful for effective patient selection and to avoid unnecessary treatment of pre-defined non-responders. This may be achieved by research focusing on the molecular mechanisms of the interaction of transdermal capsaicin with cutaneous cells and nerve fibers.

This article is based on previously conducted studies, and does not involve any new studies of human or animal subjects performed by any of the authors.

\section{ACKNOWLEDGMENTS}

No funding or sponsorship was received for this study or publication of this article. During the 
peer review process, the manufacturer of the agent under review was offered an opportunity to comment on the technical aspects of this article, and minor changes resulting from comments received were made by the author based on their scientific and editorial merit. Data are based on current scientific evidence only. Both named authors meet the ICMJE criteria for authorship for this manuscript, take responsibility for the integrity of the work as a whole, and have given final approval for the version to be published.

Compliance with ethics guidelines. This article is based on previously conducted studies and does not involve any new studies of human or animal subjects performed by any of the authors.

Conflict of interest. Nurcan Üçeyler has received travel grants and speaker honoraria from Astellas. Claudia Sommer has consulted for and received speaker honoraria from Astellas.

Open Access. This article is distributed under the terms of the Creative Commons Attribution Noncommercial License which permits any noncommercial use, distribution, and reproduction in any medium, provided the original author(s) and the source are credited.

\section{REFERENCES}

1. Treede RD, Jensen TS, Campbell JN, et al. Neuropathic pain: redefinition and a grading system for clinical and research purposes. Neurology. 2008;70:1630-5.

2. Haanpaa M, Attal N, Backonja M, et al. NeuPSIG guidelines on neuropathic pain assessment. Pain. 2011;152:14-27.

3. Baron R, Binder A, Wasner G. Neuropathic pain: diagnosis, pathophysiological mechanisms, and treatment. Lancet Neurol. 2010;9:807-19.
4. Dib-Hajj SD, Rush AM, Cummins TR, et al. Gain-offunction mutation in Nav1.7 in familial erythromelalgia induces bursting of sensory neurons. Brain. 2005;128:1847-54.

5. Tominaga M. Nociception and TRP channels. Handb Exp Pharmacol. 2007:489-505.

6. Austin PJ, Moalem-Taylor G. The neuro-immune balance in neuropathic pain: involvement of inflammatory immune cells, immune-like glial cells and cytokines. J Neuroimmunol. 2010;229:26-50.

7. Hagenacker T, Czeschik JC, Schafers M, Busselberg D. Sensitization of voltage activated calcium channel currents for capsaicin in nociceptive neurons by tumor-necrosis-factor-alpha. Brain Res Bull. 2010;81:157-63.

8. Kochukov MY, McNearney TA, Yin H, et al. Tumor necrosis factor-alpha (TNF-alpha) enhances functional thermal and chemical responses of TRP cation channels in human synoviocytes. Mol Pain. 2009;5:49.

9. Caterina MJ, Schumacher MA, Tominaga M, Rosen TA, Levine JD, Julius D. The capsaicin receptor: a heat-activated ion channel in the pain pathway. Nature. 1997;389:816-24.

10. Baron R. Pharmakologische nicht-interventionelle Therapie chronisch neuropathischer Schmerzen. In: Diener HC, Weimar C, editors. Leitlinien für Diagnostik und Therapie in der Neurologie. Stuttgart: Thieme; 2012. p. 771-83.

11. Moulin DE, Clark AJ, Gilron $\mathrm{I}$, et al. Pharmacological management of chronic neuropathic pain-consensus statement and guidelines from the Canadian Pain Society. Pain Res Manag. 2007;12:13-21.

12. Attal N, Cruccu G, Baron R, et al. EFNS guidelines on the pharmacological treatment of neuropathic pain: 2010 revision. Eur J Neurol. 2010;17:1113e1188.

13. Dworkin $\mathrm{RH}, \mathrm{O}^{\prime}$ Connor $\mathrm{AB}$, Backonja $\mathrm{M}$, et al. Pharmacologic management of neuropathic pain: evidence-based recommendations. Pain. 2007;132:237-51.

14. Jensen TS, Madsen CS, Finnerup NB. Pharmacology and treatment of neuropathic pains. Curr Opin Neurol. 2009;22:467-74.

15. Harden $\mathrm{N}$, Cohen $\mathrm{M}$. Unmet needs in the management of neuropathic pain. J Pain Symptom Manag. 2003;25:S12-7.

16. Treede RD, Wagner T, Kern KU, et al. Mechanismand experience-based strategies to optimize 
treatment response to the capsaicin $8 \%$ cutaneous patch in patients with localized neuropathic pain. Curr Med Res Opin. 2013;29:527-38.

17. Simone DA, Nolano M, Johnson T, WendelschaferCrabb G, Kennedy WR. Intradermal injection of capsaicin in humans produces degeneration and subsequent reinnervation of epidermal nerve fibers: correlation with sensory function. J Neurosci. 1998;18:8947-59.

18. Kennedy WR, Vanhove GF, Lu SP, et al. A randomized, controlled, open-label study of the long-term effects of NGX-4010, a highconcentration capsaicin patch, on epidermal nerve fiber density and sensory function in healthy volunteers. J Pain. 2010;11:579-87.

19. Goswami C, Schmidt H, Hucho F. TRPV1 at nerve endings regulates growth cone morphology and movement through cytoskeleton reorganization. FEBS J. 2007;274:760-72.

20. Devigili G, Tugnoli V, Penza P, et al. The diagnostic criteria for small fibre neuropathy: from symptoms to neuropathology. Brain. 2008;131:1912-25.

21. Petersen KL, Rice FL, Farhadi $M$, Reda $H$, Rowbotham MC. Natural history of cutaneous innervation following herpes zoster. Pain. 2010;150:75-82.

22. Ji RR, Samad TA, Jin SX, Schmoll R, Woolf CJ. p38 MAPK activation by NGF in primary sensory neurons after inflammation increases TRPV1 levels and maintains heat hyperalgesia. Neuron. 2002;36:57-68.

23. Baron R. Peripheral neuropathic pain: from mechanisms to symptoms. Clin J Pain. 2000;16: S12-20.

24. Bowles WR, Sabino M, Harding-Rose C, Hargreaves KM. Chronic nerve growth factor administration increases the peripheral exocytotic activity of capsaicin-sensitive cutaneous neurons. Neurosci Lett. 2006;403:305-8.

25. Kim JH, Ko JA, Kim JT, et al. Preparation of a capsaicinloaded nanoemulsion for improving skin penetration. J Agric Food Chem. 2014 [Epub ahead of Print].

26. Ständer S, Moormann C, Schumacher M, et al. Expression of vanilloid receptor subtype 1 in cutaneous sensory nerve fibers, mast cells, and epithelial cells of appendage structures. Exp Dermatol. 2004;13:129-39.

27. Acorda Therapeutics, Inc. Qutenza: Treatment Guide. Available from: http://www.qutenza.com/_ docs/Qutenza\%20Treatment\%20Guide.pdf. Accessed April 14, 2014.
28. Committee for Medicinal Products for Human Use (CHMP). European Public Assessment ReportQutenza. Available from: www.ema.europa.eu/ docs/en_GB/document_library/EPAR_-_Product_ Information/human/000909/WC500040453.pdf. Accessed July 15, 2014.

29. Backonja M, Wallace MS, Blonsky ER, et al. NGX4010, a high-concentration capsaicin patch, for the treatment of postherpetic neuralgia: a randomised, double-blind study. Lancet Neurol. 2008;7: 1106-12.

30. Backonja MM, Malan TP, Vanhove GF, Tobias JK, Group CS. NGX-4010, a high-concentration capsaicin patch, for the treatment of postherpetic neuralgia: a randomized, double-blind, controlled study with an open-label extension. Pain Med. 2010;11:600-8.

31. Irving GA, Backonja MM, Dunteman E, et al. A multicenter, randomized, double-blind, controlled study of NGX-4010, a high-concentration capsaicin patch, for the treatment of postherpetic neuralgia. Pain Med. 2011;12:99-109.

32. Webster LR, Malan TP, Tuchman MM, Mollen MD, Tobias JK, Vanhove GF. A multicenter, randomized, double-blind, controlled dose finding study of NGX-4010, a high-concentration capsaicin patch, for the treatment of postherpetic neuralgia. J Pain. 2010;11:972-82.

33. Clifford DB, Simpson DM, Brown S, et al. A randomized, double-blind, controlled study of NGX-4010, a capsaicin 8\% dermal patch, for the treatment of painful HIV-associated distal sensory polyneuropathy. J Acquir Immune Defic Syndr. 2012;59:126-33.

34. Simpson DM, Brown S, Tobias J, Group N-CS. Controlled trial of high-concentration capsaicin patch for treatment of painful HIV neuropathy. Neurology. 2008;70:2305-13.

35. Derry S, Sven-Rice A, Cole P, Tan T, Moore RA. Topical capsaicin (high concentration) for chronic neuropathic pain in adults. The Cochrane Database of Systematic Reviews. 2013;2:CD007393.

36. Clinicaltrials.gov. Method of Pre-treatment for Application of QUTENZA Capsaicin 8\% Patch (LIFT). Available from: http://clinicaltrials.gov/ct2/ show/record/NCT01416116. Accessed April 14, 2014

37. Mou J, Paillard F, Turnbull B, Trudeau J, Stoker M, Katz NP. Efficacy of Qutenza(R) (capsaicin) 8\% patch for neuropathic pain: a meta-analysis of the Qutenza Clinical Trials Database. Pain. 2013;154: 1632-9. 
38. Mou J, Paillard F, Turnbull B, Trudeau J, Stoker M, Katz NP. Qutenza (Capsaicin) 8\% patch onset and duration of response and effects of multiple treatments in neuropathic pain patients. Clin J Pain. 2014;30:286-94.

39. Maihöfner C, Heskamp ML. Prospective, noninterventional study on the tolerability and analgesic effectiveness over 12 weeks after a single application of capsaicin 8\% cutaneous patch in 1,044 patients with peripheral neuropathic pain: first results of the QUEPP study. Curr Med Res Opin. 2013;29:673-83.

40. Brown S, Simpson DM, Moyle G, et al. NGX-4010, a capsaicin $8 \%$ patch, for the treatment of painful
HIV-associated distal sensory polyneuropathy: integrated analysis of two phase III, randomized, controlled trials. AIDS Res Ther. 2013;10:5.

41. Maihöfner CG, Heskamp ML. Treatment of peripheral neuropathic pain by topical capsaicin: impact of pre-existing pain in the QUEPP-study. Eur J Pain. 2014;18:671-9.

42. Martini $\mathrm{CH}$, Yassen A, Krebs-Brown A, et al. A novel approach to identify responder subgroups and predictors of response to low- and high-dose capsaicin patches in postherpetic neuralgia. Eur J Pain. 2013;17:1491-501. 